\title{
The Role of Heart Rate Variability and Fragmented QRS for Determination of Subclinical Cardiac Involvement in Beta-Thalassemia Major
}

\author{
Mozhgan Parsaee $^{a} \quad$ Amir Farjam Fazelifar $^{\mathrm{b}} \quad$ Elham Ansaripour $^{\mathrm{b}}$ \\ Azita Azarkeyvan $^{c}$ Behshid Ghadrdoost ${ }^{d}$ Ashraf Charmizadeh $^{d}$ \\ Mohaddeseh Behjatia \\ ${ }^{a}$ Echocardiography Research Center, Rajaie Cardiovascular Medical and Research Center, \\ Iran University of Medical Sciences, Tehran, Iran; ${ }^{\text {b } C a r d i a c ~ E l e c t r o p h y s i o l o g y ~ R e s e a r c h ~}$ \\ Center, Rajaie Cardiovascular Medical and Research Center, Iran University of Medical \\ Sciences, Tehran, Iran; ${ }^{C}$ Research Center of Iranian Blood Transfusion Organization, \\ Thalassemia Clinic, Tehran, Iran; d Rajaie Cardiovascular Medical and Research Center, Iran \\ University of Medical Sciences, Tehran, Iran
}

\section{Keywords}

Thalassemia major · Subclinical cardiac dysfunction · Cardiac T2* · Fragmented QRS · Holter monitoring

\begin{abstract}
Background: Iron accumulation leads to increased susceptibility to cardiovascular diseases in thalassemia major (TM) patients. Depressed heart rate variability (HRV) and development of arrhythmia are among the manifestations of subclinical cardiac involvement in TM cases. Determination of subclinical cardiac involvement is essential for preventive measures. Thus, we aimed to evaluate the best method for identification of subclinical cardiac dysfunction in TM cases. Materials and Methods: In this prospective study, 45 TM and 45 non-TM cases, who were referred for cardiac evaluation, were enrolled. Exclusion criteria included non-sinus rhythm and overt cardiac disease. TM cases underwent cardiac MRI, electrocardiography (ECG), and Holter monitoring. TM cases were divided into two groups of normal versus iron overload with a cardiac T2* of more or less than 20 ms, respectively. The non-TM cases underwent only ECG and Holter monitoring. Results: We observed no significant difference regarding HRV between normal versus iron overload TM and non-TM cases. Higher rates of premature atrial complex, low limb voltage, low atrial rhythm, as well as minimum and average HR with lower QRS duration and PR interval were detected in TM versus non-TM cases ( $p$ value $<0.05$ ). Conclusions: We observed a higher prevalence of low limb voltage and low
\end{abstract}


atrial rhythm in TM cases versus non-TM cases. Indeed, the role of fragmented QRS (fQRS) for subclinical detection of cardiac disease in TM cases is still so controversial and needs more evaluation. Application of HRV and $\mathrm{fQRS}$ in this regard may need to be performed at the right time point after initiation of blood transfusion, but this needs to be determined.

(C) 2020 S. Karger AG, Basel

\section{Introduction}

Thalassemia major (TM) is an inherited hemoglobin disorder with subsequent chronic hemolytic anemia due to the absent synthesis of globin chains essential for hemoglobin formation [1]. This disease state needs regular long-life blood transfusion and iron chelation therapy for proper survival [2]. Iron accumulation leads to the development of cardiac siderosis which results in occurrence of heart failure and arrhythmias which are the major cause of mortality in TM patients [3]. Thus, determination of subclinical cardiac involvement in thalassemia is essential for preventive therapeutic measures.

One of the manifestations of subclinical cardiac involvement in TM is arrhythmia, and various electrocardiography (ECG) alterations have been reported in TM patients without heart failure [4]. Susceptibility to arrhythmia could be detected by Holter monitoring and fragmented QRS (fQRS). Several investigations have shown the relationship between presence of $\mathrm{fQRS}$, as a marker of depolarization abnormality and subsequent predisposition to arrhythmia, with cardiac iron overload detected by CMR in TM cases [5]. Depressed heart rate variability (HRV) detected by 24-h Holter monitoring, as an indicator of cardiac autonomic function, could be used for earlier diagnosis of iron overload cardiomyopathy in TM cases [6].

It is well known that CMR-T2* is the gold standard technique and a powerful tool for both earlier detections of myocardial siderosis and monitoring of cardiac iron content and detection of subtle changes of the left ventricular ejection fraction [7]. By now, various direct and indirect methods have been assessed for an earlier diagnosis of cardiac iron overload in TM patients such as echocardiogram, serum ferritin level, HRV, CMR-T2*, as well as liver and cardiac biopsy [8]. But the results are controversial. The optimal test for earlier detection of cardiac overload should be fast, noninvasive, inexpensive, and widely accessible. In many centers that provide care for TM patients, CMR accessibility is limited and patients need to be referred to other centers that have limited proper patient follow-up. Therefore, in this study, we aimed to evaluate the relationship between Holter monitoring, fQRS, and CMR for determination of subclinical cardiac involvement in TM patients.

\section{Materials and Methods}

In this prospective study, $45 \mathrm{TM}$ cases with an age range between 17 and 50 years and a history of blood transfusion, who were referred to our center for evaluation of cardiac function with or without history of palpitation, were enrolled. Exclusion criteria included cases without sinus rhythm and presence of overt cardiac disease manifested as reduced ejection fraction, valvular heart disease, and pulmonary arterial hypertension. Participants should have received blood transfusion within the past week before inclusion. As control group, 45 healthy non-TM cases in sinus rhythm who were referred only for evaluation of palpitation were also included. We were blinded to cardiac iron loading status of the TM participants. Participants were interviewed and data regarding gender, age, cardiovascular risk factors, blood transfusion interval, and the type of chelation therapy were obtained using a prepared structured questionnaire.

Included TM cases underwent cardiac MRI for evaluation of T2* measures, 12-lead surface ECG, and 24-h Holter monitoring. We evaluated the RP interval, QRS duration, QT interval, premature atrial and ventricular complexes, low atrial rhythm, pause more than $3 \mathrm{~ms}$, sinus arrhythmia, sinus tachycardia, sinus bradycardia, wandering pacemaker, various forms of atrio-ventricular block, low limb voltage, transitional 
Parsaee et al:: Holter Monitoring and Fragmented QRS in Beta-Thalassemia Major

Table 1. Demographic and clinical characteristics of all patients

\begin{tabular}{lc}
\hline Variables & $n(\%)$ \\
\hline Mean age \pm SD, years & $31.96 \pm 7.5$ \\
Male & $20(44.4)$ \\
Diabetes mellitus & $13(28.9)$ \\
Smoking & $3(6.7)$ \\
Hypothyroidism & $5(11.1)$ \\
Using combined oral and subcutaneous $\quad$ \\
$\quad$ chelation therapy & $22(48.8)$ \\
Splenectomy & $20(44.4)$ \\
Aspirin therapy & $17(37.8)$ \\
Mean hemoglobin \pm SD, $\mathrm{mg} / \mathrm{dL}$ & $9.58 \pm 1.39$ \\
Mean serum ferritin \pm SD, $\mathrm{mg} / \mathrm{dL}$ & $2,318.74 \pm 2,099$ \\
\hline
\end{tabular}

zone, sustained and nonsustained supraventricular tachycardia, minimal heart rate (HR), maximal HR, average HR, as well as HRV and ECG criteria of RV dysfunction. fQRS was defined by the presence of notching of R or S wave or R' wave in the presence of narrow QRS. We performed HRV using time domain measures, in which HR at any intervals between successive normal complexes or any point at time are determined. Each QRS complex in a continuous ECG record is detected, and all intervals between adjacent QRS complexes after sinus node depolarizations (normal-to-normal or NN intervals) or instantaneous HR are determined. The mean HR, the mean NN interval, the difference between day and night HR, and the difference between shortest and longest NN intervals were calculated using simple time domain measurement. All tests were mostly performed at the same day or maximally within 3 days of patient referral. Lab data including hemoglobin and the serum ferritin level were also included.

\section{Statistics}

Statistical analysis was performed with SPSS 15 for Windows (SPSS Inc., Chicago, IL, USA). Mean standard deviation (SD) and frequency were used as descriptive analysis. For statistical analysis, we evaluated once the desired parameters in TM patients in two groups based on cardiac T2* $<20$ ms considered as cases with cardiac iron overload versus cardiac T2*s $>20 \mathrm{~ms}$ considered as cases without cardiac iron overload. Once again, these parameters were compared in TM cases versus non-TM cases. Holter and surface ECG-derived data were all evaluated and compared both within the TM group, within the non-TM group and between the TM versus non-TM groups. For evaluation of the distribution of data, the one-sample KolmogorovSmirnov test was used. To compare the mean variables between the two groups, an independent $t$ test or Mann-Whitney U test was used. Qualitative data were compared with the $\chi^{2}$ test or Kruskal Wallis test (ordinal variables). To compare changes in stress echocardiography parameters during the study, paired $t$ test or Wilcoxon test was used. A $p$ value $<0.05$ was considered statistically significant.

\section{Results}

Demographic and clinical characteristics of all patients are shown in Table 1 . The serum ferritin level was significantly higher in TM cases with lower cardiac T2* $(3,288.56 \pm 2,119$ $\mathrm{mg} / \mathrm{dL})$ versus higher cardiac T2* $(1,542.90 \pm 1,763 \mathrm{mg} / \mathrm{dL})(p$ value $=0.001)$. The serum ferritin level was significantly related with severe and very severe hepatic iron overload $(p$ value $=0.043$ ). The percentage of no, mild, moderate, severe, and very severe hepatic iron overload in TM patients was $26.7,17.8,15.6,22.2$, and $17.8 \%$, respectively, and very severe hepatic iron overload was significantly related to a cardiac T2* $<20 \mathrm{~ms}$ ( $p$ value $=0.29$ ). The percentage of no, mild, moderate, and severe cardiac iron overload in TM patients was 57.8, $17.8,4.4$, and $20 \%$, respectively. Generally, the percentage of cardiac iron overload was 44 versus 55\% for noncardiac iron overload. The type of chelation therapy had no relationship with any other data. 
Table 2. ECG parameters in thalassemia versus non-thalassemia cases

\begin{tabular}{lccc}
\hline Parameter & Thalassemia & Non-Thalassemia & $p$ value \\
\hline Average HR, bpm & $85.78 \pm 11.78$ & $77.07 \pm 12.79$ & 0.001 \\
Minimum HR, bpm & $59.15 \pm 9.99$ & $53.29 \pm 12.96$ & 0.001 \\
Maximum HR, bpm & $131.83 \pm 19.84$ & $124.16 \pm 26.41$ & 0.064 \\
QTc, ms & $401.40 \pm 45.51$ & $406.56 \pm 15.10$ & 0.478 \\
QRS duration, ms & $44 \pm 13.888$ & $88.89 \pm 11.22$ & 0.000 \\
PR interval, ms & $163.07 \pm 44$ & $155.67 \pm 17.50$ & 0.029 \\
\hline
\end{tabular}

Table 3. ECG abnormality in thalassemia versus non-thalassemia cases

\begin{tabular}{lccl}
\hline Parameter & Thalassemia & Non-Thalassemia & $p$ value \\
\hline NSSVT & $33.3 \%$ & $20 \%$ & 0.166 \\
Low limb voltage & $9.1 \%$ & $0.0 \%$ & 0.038 \\
Low atrial rhythm & $4 \%$ & $0.0 \%$ & 0.03 \\
Abnormal HRV & $2.6 \%$ & $2.2 \%$ & 0.90 \\
PAC & & & \\
$\quad$ No & $17(37.8)$ & $6(15.4)$ & \\
$\quad<1 \%$ & $28(62.2)$ & $30(76.9)$ & \\
$1-10 \%$ & $0(0)$ & $2(5.1)$ & \\
$\quad>10 \%$ & $0(0)$ & $1(2.6)$ & \\
PVC & & & \\
$\quad$ No & $22(48.9)$ & $16(41)$ & 0.412 \\
$<1 \%$ & $22(48.9)$ & $21(53.8)$ & \\
$1-10 \%$ & $1(2.2)$ & $2(5.1)$ & \\
\hline
\end{tabular}

NSSVT, nonsustained supraventricular tachycardia; HRV, heart rate variability; PAC, premature atrial complex; PVC, premature ventricular complex.

The minimum and average HR were significantly higher in TM versus non-TM cases ( $p$ value $=0.001$ for both). QRS duration and PR interval were significantly lower in TM versus non-TM cases ( $p$ value $=0.000$ and 0.029 , respectively) (Table 2 ). The PAC rate was significantly higher in TM versus non-TM cases ( $p$ value $=0.008$ ), but the PVC rate did not differ between the groups. TM cases had a significantly higher frequency of low limb voltage $(9.1 \%)$ versus non-TM cases $(0 \%)$ ( $p$ value $=0.038)$. There was no difference in the rate of NSSVT, fQRS, HRV, transitional zone, and RV dysfunction detected by ECG criteria between TM and non-TM cases ( $p$ value $>0.05$ ). The frequency of low atrial rhythm was significantly higher in TM cases $(90 \%)$ versus non-TM cases $(0 \%)(p$ value $=0.03)($ Table 3$)$.

\section{Discussion}

Various forms of arrhythmia have been observed in iron overload conditions based on the duration of iron deposition. TM patients with arrhythmia symptoms are potentially at increased risk of mortality [9]. Arrhythmia is one of the cardinal cardiac manifestations in TM cases with iron overload state [10]. The pathophysiologic mechanisms for higher susceptibility to arrhythmia in TM cases with iron overload state has been attributed to interference of accumulated iron with cardiac electrical function, free radicals, myocardial fibrosis, and apoptosis [11]. However, we observed no significant differences regarding arrhythmia or 
parameters of surface ECG and Holter monitoring between iron overload versus nonoverload TM cases. We suggest that the duration of iron overload state and the efficacy of iron chelation strategies might be involved in the occurrence of arrhythmia in this setting.

As in our findings, the significantly increased arrhythmia burden has been reported in TM cases without overt cardiomyopathy [4]. The higher minimum and average HR as well as shorter QRS duration and PR interval in TM versus non-TM cases could be explained by the anemia state and compensatory tachycardia [12]. The higher rate of PAC in TM versus non-TM cases could also be explained by anemia. We observed a higher frequency of low limb voltage in TM versus non-TM cases. This ECG finding could be seen in TM cases with heart failure but is uncommon in TM cases without heart failure [13]. To date, we have no definite explanation for the higher prevalence of low atrial rhythm in TM versus non-TM cases, and this arrhythmia has not been reported as a frequent complication in TM cases. This might be due to the higher deposition of iron in the sinoatrial node of TM cases with iron overload, but in our study, the prevalence of low atrial rhythm was not different between TM cases with or without iron overload. We suggest an evaluation of this hypothesis by using CMR with the tissue characterization imaging modality.

HRV which reflects an autonomic function has been reported to be depressed in TM cases with cardiac iron overload [8]. There are some suggestions about a possible lack of correlation between HRV and iron overload which is the same as in our findings [5]. The presence of fQRS on surface ECG has been seen in parallel with decreased cardiac T2* [5]. The less frequent presence of fQRS in TM cases under treatment with deferasirox has been reported previously [14]. The absence of fQRS in surface ECG in our study even in TM cases with iron overload may be due to the early stage of disease. Therefore, the controversial role of fQRS and HRV is a further obstacle for its universal applicability in earlier diagnosis of subclinical cardiac disease in TM cases.

\section{Conclusions}

We observed a higher prevalence of low limb voltage and low atrial rhythm in TM cases compared with non-TM cases. To date, we have no explanation for this finding. Indeed, the role of fQRS for subclinical detection of cardiac disease in TM cases is still so controversial and needs more evaluation. The application of HRV and fQRS in this regard may need to be performed at the right time point after initiation of blood transfusion, but this needs to be determined.

\section{Limitations of the Study}

Our data needs further confirmation by studies with more sample volume. Low sample size is the limitation of our study.

\section{Acknowledgement}

We would like to thank all cardiology staff in Rajaie cardiovascular research center of assisting with providing this manuscript.

\section{Statement of Ethics}

This study was approved by our local ethical committee according to the Helsinki Declaration of the World Medical Association (2000). All participants provided signed informed consent prior to study initiation.

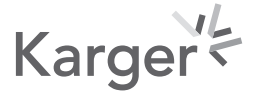




\begin{tabular}{l|l}
\hline Pulse 2020;8:15-20 \\
\hline DOI: 10.1159/000505556 & $\begin{array}{l}\text { @ 2020 S. Karger AG, Basel } \\
\text { www.karger.com/pls }\end{array}$ \\
\hline
\end{tabular}

\section{Disclosure Statement}

The authors have no conflicts of interest to declare.

\section{Funding Sources}

This research did not receive any specific grant from funding agencies in the public, commercial, or notfor-profit sectors.

\section{Author Contributions}

Conducting the project study, final approval of the article: Mohaddeseh Behjati and Mozhgan Parsaee. Conception and design, as well as management of the article: Mozhgan Parsaee and Amir Farjam Fazelifar. Collecting the data and preparing the manuscript: Elham Ansaripour, Azita Azarkeyvan, Ashraf Charmizadeh, Behshid Ghadrdoost, Mozhgan Parsaee, and Mohaddeseh Behjati. Analysis: Behshid Ghadrdoost.

\section{References}

1 Russo V, Rago A, Papa AA, Nigro G. Electrocardiographic Presentation, Cardiac Arrhythmias, and Their Management in $\beta$-Thalassemia Major Patients. Ann Noninvasive Electrocardiol. 2016 Jul;21(4):335-42.

2 Kumfu S, Fucharoen S, Chattipakorn SC, Chattipakorn N. Cardiac complications in beta-thalassemia: from mice to men. Exp Biol Med (Maywood). 2017 Jun;242(11):1126-35.

3 Franzoni F, Galetta F, Di Muro C, Buti G, Pentimone F, Santoro G. Heart rate variability and ventricular late potentials in $\beta$-thalassemia major. Haematologica. 2004 Feb;89(2):233-4.

4 Kolios M, Korantzopoulos P, Vlahos AP, Kapsali E, Briasoulis E, Goudevenos JA. Electrocardiographic abnormalities and arrhythmic risk markers in adult patients with beta thalassemia major. Int J Cardiol. 2016 Oct; 221:932-6.

5 Bayar N, Kurtoğlu E, Arslan Ş, Erkal Z, Çay S, Çağırcı G, et al. Assessment of the relationship between fragmented QRS and cardiac iron overload in patients with beta-thalassemia major. Anatol J Cardiol. 2015 Feb; 15(2):132-6.

6 Wijarnpreecha K, Siri-Angkul N, Shinlapawittayatorn K, Charoenkwan P, Silvilairat S, Siwasomboon C, et al. Heart Rate Variability as an Alternative Indicator for Identifying Cardiac Iron Status in Non-Transfusion Dependent Thalassemia Patients. PLoS One. 2015 Jun;10(6):e0130837.

7 Cappellini MD, Cohen A, Porter J, Taher A, Viprakasit V. Guidelines for the Management of Transfusion Dependent Thalassaemia (TDT) [Internet]. 3rd edition. Nicosia (CY): Thalassaemia International Federation; 2014.

8 Koonrungsesomboon N, Chattipakorn SC, Fucharoen S, Chattipakorn N. Early detection of cardiac involvement in thalassemia: from bench to bedside perspective. World J Cardiol. 2013 Aug; 5(8):270-9.

9 Kremastinos DT, Tsetsos GA, Tsiapras DP, Karavolias GK, Ladis VA, Kattamis CA. Heart failure in beta thalassemia: a 5-year follow-up study. Am J Med. 2001 Oct;111(5):349-54.

10 Isma'eel H, Cappellini MD, Taher A. Chronic transfusion, iron overload and cardiac dysfunction: a multi-dimensional perspective. Br J Cardiol. 2008;15:40-5.

11 Kayrak M, Acar K, Gul EE, Ozbek O, Abdulhalikov T, Sonmez 0, et al. The Association between Myocardial Iron Load and Ventricular Repolarization Parameters in Asymptomatic Beta-Thalassemia Patients. Adv Hematol. 2012;2012:170510.

12 Detterich J, Noetzli L, Dorey F, Bar-Cohen Y, Harmatz P, Coates T, et al. Electrocardiographic consequences of cardiac iron overload in thalassemia major. Am J Hematol. 2012 Feb;87(2):139-44.

13 Mancuso L, Mancuso A, Bevacqua E, Rigano P. Electrocardiographic abnormalities in thalassemia patients with heart failure. Cardiovasc Hematol Disord Drug Targets. 2009 Mar; 9(1):29-35.

14 Karakulak UN, Tutkun E, Yllmaz ÖH. Iron overload and fragmented QRS in patients with Thalassemia major: Mechanisms, therapies, and new horizons. Anatol J Cardiol. 2015 Jul;15(7):592-3. 